\title{
The anion-transfer system of erythrocyte membranes
}

\author{
$N$-(7-Nitrobenzofurazan-4-yl)taurine, a fluorescent substrate-analogue of the system
}

\author{
Ofer EIDELMAN. Marina ZANGVILL, Michal RAZIN, Hagai GINSBURG and \\ Z. Ioav CABANTCHIK \\ Department of Biological Chemistry, The Hebrew University of Jerusalem, Institute of Life Sciences, \\ Jerusalem, Israel 91904
}

(Received 12 January 1981/Accepted 3 February 1981)

\begin{abstract}
The fluorescent probe Nbd-Tau [ $N$-(7-nitrobenzofurazan-4-yl)taurine] was synthesized and evaluated as a potential substrate of the anion-transport system of human erythrocyte membranes. The probe inhibited $\mathrm{Cl}^{-}$exchange in a competitive manner from either surface of the membrane, displaying $K_{1}$ values in the mM range at the inner surface and in the $\mu \mathrm{M}$ range at the outer surface. Inhibition from within cells was via interaction with $\mathrm{Cl}^{-}$-transport sites, whereas from without it was via interaction with sites of unidentified nature. Nbd-Tau efflux from cells was monitored fluorimetrically in a continuous mode by a novel method that circumvents separation of the cells from the medium. Using this method, it is shown that Nbd-Tau efflux fulfils the following criteria of a substrate of the anion transport system: (a) susceptibility to classical and specific inhibitors of the system; (b) competitive inhibition with $\mathrm{Cl}^{-}$for anion-transport sites; and (c) temperature coefficient comparable with that of $\mathrm{Cl}^{-}$exchange. The fluorometric method is highly sensitive, versatile, and kinetically informative. With minor modifications it can be used for measuring anion transport across 'ghost' and isolated membrane vesicles.
\end{abstract}

The anion transport system of human erythrocytes has been extensively studied in terms of kinetic properties (Cabantchik et al., 1978; Knauf, 1979; Gunn, 1979), physiological relevance (Wieth \& Brahm, 1980), and structural organization of the functional polypeptides in the membrane matrix (Cabantchik et al., 1978; Steck, 1978; Tanner, 1978; Guidotti, 1980). Various attempts have also been made at its functional isolation (Wolosin et al., 1977) and reconstitution (Rothstein et al., 1975; Ross \& McConnel, 1978; Cabantchik et al., 1980; Cabantchik \& Loyter, 1980). However, major technical difficulties were found in studying transport in reconstituted systems, which were due to the rapidity of fluxes that take place in systems of high surface to volume ratios (e.g., vesicles) and the need for quick and efficient separation of vesicles from medium at defined times. Furthermore, there were limitations in the use of radioactive labelled substrates in terms of available specific activities relative to the small trapped volume of vesicles.

Abbreviations used: Nbd-Tau, $N$-(7-nitrobenzofurazan-4-yl)taurine; Nbd-Cl, 4-chloro-7-nitrobenzofurazan; DNDS, 4,4'-dinitro-2,2'-stilbenedisulphonic acid.
In an attempt to provide a sensitive marker for the activity of the red blood cell anion transporter, we developed a method of continuous monitoring of transport by fluarescence (Eidelman \& Cabantchik, 1980). The method relies on the fluorescent anion analogue Nbd-Tau (Fig. 1) whose excitation and emission spectra overlap substantially with the absorption spectrum of haemoglobin. The selection of Nbd-Tau as a potential substrate was based primarily on our previous observations that the 2-nitro-4-azidophenyl derivative of taurine can serve as substrate of the system (Cabantchik et al., 1976; Knauf et al., 1978) and on structure-activity considerations of

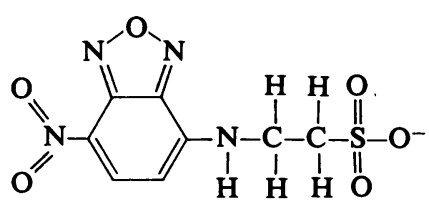

Nbd-Tau

Fig. 1. Chemical structure of Nbd-Tau 
potential anion-transport inhibitors (Barzilay et al., 1979). As fluorescence originating from intracellular Nbd-Tau is mostly quenched, the egress of the analogue could be followed fluorimetrically in a continuous fashion, circumventing the need for separating cells from medium. In this work we demonstrate that Nbd-Tau can serve specifically as a substrate of the anion transport system of human erythrocytes. We base this claim on the following criteria: mutual competition between $\mathrm{Cl}^{-}$and $\mathrm{Nbd}-$ Tau for transport sites, common susceptibility to specific inhibitors of the system, and similar temperature dependence. The method was sensitive with respect to time as well as with respect to the number of cells required, versatile (inasmuch as various systems can be monitored in parallel in an environmentally controlled fashion) and kinetically informative. The method used here to measure anion transport across intact erythrocytes was also adapted for studying anion transfer across white 'ghosts' or vesicles by replacing haemoglobin with a complexation quencher of Nbd-Tau (Darmon et al., 1980).

\section{Materials and methods}

\section{Source of materials}

Chemicals were obtained from the following sources: Nbd-Cl, from Molecular Probes, Plano, TX, U.S.A.; phloretin and DNDS from ICN (K \& K) Chemicals; 2-aminoethanesulphonic acid (taurine) from Sigma; Amphotericin B (type 1) from Squibb \& Sons, Inc. All other chemicals were of either analytical or the best available grade. The covalent reagent $4,4^{\prime}$-di-isothiocyano- $2,2^{\prime}$ 'stilbenedisulphonic acid was prepared and used as previously described (Cabantchik \& Rothstein, 1972).

\section{Synthesis and properties of Nbd-Tau}

A solution of taurine $(0.4 \mathrm{M})$ in $\mathrm{Na}_{2} \mathrm{CO}_{3}(1 \mathrm{M}$, $10 \mathrm{ml})$ was treated with $\mathrm{Nbd}-\mathrm{Cl}(0.5 \mathrm{M})$ in ethanol $(10 \mathrm{ml})$ for $120 \mathrm{~min}$ at $45^{\circ} \mathrm{C}$. The product was evaporated to dryness, extracted with diethyl ether, dissolved in distilled water, and dialysed against distilled water at $4^{\circ} \mathrm{C}$ (Spectrapor-2 dialysis bag) to eliminate polymerized material. The diffusate was evaporated to dryness and extracted with ethyl acetate in a Soxhelet apparatus. The residue, which consisted of the sodium salt of Nbd-Tau, was extracted with ethanol in the same apparatus and crystallized from ethanol overnight at $-10^{\circ} \mathrm{C}$. The acid form of Nbd-Tau was obtained by dissolving the sodium salt in water and passing the solution over Dowex 50W $\left(\mathrm{H}^{+}\right.$form) resin. The solution was evaporated to dryness, washed with diethyl ether and subsequently with light petroleum (b.p. $60-80^{\circ} \mathrm{C}$ ), and kept dessicated at $-10^{\circ} \mathrm{C}$. The final yield was $65 \%$.

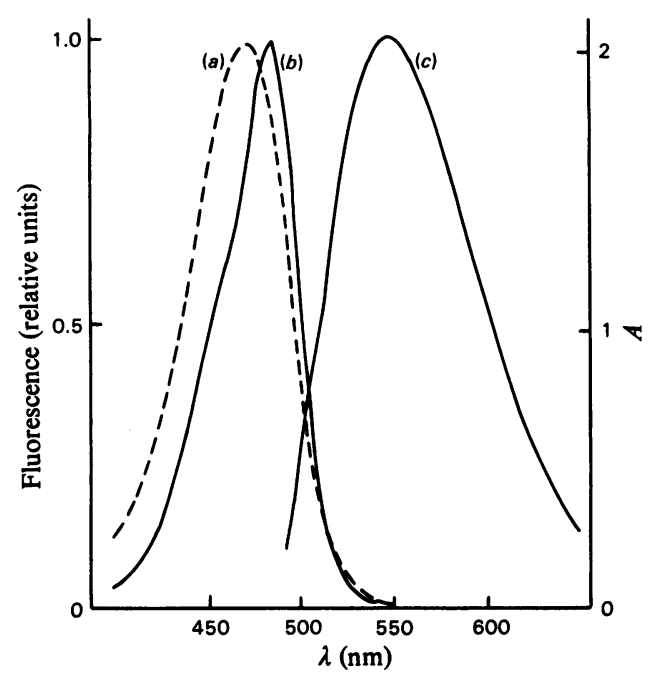

Fig. 2. Spectral properties of Nbd-Tau

Excitation (b) and emission (c) spectra of Nbd-Tau $(0.1 \mu \mathrm{M})$ in phosphate-buffered saline $\left(\mathrm{pH} 7.4,25^{\circ} \mathrm{C}\right)$ are shown in full lines. The absorption spectrum of the compound $(100 \mu \mathrm{M})$ in the same medium is shown (a) (broken lines).

The new compound had $\lambda_{\max }$ in aqueous solution (pH4-8) $473 \mathrm{~nm}\left(\varepsilon=23200\right.$ litre $\left.\cdot \mathrm{mol}^{-1} \cdot \mathrm{cm}^{-1}\right)$, a fluorescence excitation maximum at $483 \mathrm{~nm}$ and a fluorescence emission maximum at $550 \mathrm{~nm}$ (Fig. 2). (Found: C, 28.2; H, 3.25; N, 16.5; S, 9.8; loss at $100^{\circ} \mathrm{C}, 10$. Calc. for $\mathrm{C}_{8} \mathrm{H}_{8} \mathrm{~N}_{4} \mathrm{~S}_{1}, 2 \mathrm{H}_{2} \mathrm{O}: \mathrm{C}, 29.4 ; \mathrm{H}$, 3.7: $\left.\mathrm{N}, 17.1 ; \mathrm{S}, 9.8 ; \mathrm{H}_{2} \mathrm{O}, 11 \%\right)$. The compound gave a single spot on t.l.c. plates, showing an $R_{F}$ of 0.75 in propan-1-ol/ $\mathrm{NH}_{4} \mathrm{OH} /$ water $(6: 3: 2$, by vol.) and an $R_{F}$ of 0.2 in butan-1-ol saturated with water.

\section{Spectroscopic techniques}

Absorption spectra were determined with a Gilford spectrophotometer. Fluorescence excitation and emission spectra and time-dependent changes in fluorescence were monitored in a Perkin-Elmer MPF-4 spectrofluorimeter equipped wtih a fourcuvette turret and a thermostated unit connected to a constant-temperature bath-circulator (Haake).

Preparation of erythrocytes at different $\mathrm{Cl}^{-}$concentrations

Erythrocytes withdrawn from healthy donors into heparinized tubes, or from recently outdated blood obtained from a blood bank (Hadassah Medical Center), were washed several times in iso-osmotic phosphate-buffered saline $\left(\mathrm{NaH}_{2} \mathrm{PO}_{4}, 20 \mathrm{mM} ; \mathrm{NaCl}\right.$, $150 \mathrm{~mm}$; titrated wtih $\mathrm{NaOH}$ to $\mathrm{pH} 7.4$, or as indicated) to remove the buffy coat. The cell $\mathrm{Cl}^{-}$ concentration was modified by varying the extra- 
cellular $\mathrm{Cl}^{-}$concentration in the presence of Amphotericin B. This method is a modification (Schnell et al., 1977) of the Nystatin technique described by Dalmark (1976). Briefly, cells (10\% haematocrit) were washed with solutions containing $100-500 \mathrm{~mm}$ $\mathrm{KCl} / 25 \mathrm{~mm}-\mathrm{KH}_{2} \mathrm{PO}_{4}$ [titrated to $\mathrm{pH} 7.0$ (or as indicated) $] / 30 \mathrm{~mm}$-sucrose and $5 \mu \mathrm{g}$ of Amphotericin $\mathrm{B} / \mathrm{ml}$, and incubated in this medium for $90 \mathrm{~min}$ at $37^{\circ} \mathrm{C}$. For solutions containing concentrations of $\mathrm{KCl}$ lower than $100 \mathrm{mM}$, cells were washed first with $150 \mathrm{mM}-\mathrm{KCl}$ in the presence of Amphotericin B and subsequently diluted by slow addition of a $25 \mathrm{~mm}-\mathrm{KH}_{2} \mathrm{PO}_{4} / 30 \mathrm{~mm}$-sucrose solution ( $\mathrm{pH}$ 7.0) until the final $\mathrm{Cl}^{-}$concentration was reached. The $\mathrm{Cl}^{-}$-loaded cells were washed thrice with the appropriate medium (same $\mathrm{Cl}^{-}$concentration as reached in the cells) and incubated for another hour in the same medium.

\section{Uptake of Nbd-Tau by erythrocytes}

For measurements of the time dependence of Nbd-Tau uptake, cells ( $10 \%$ haematocrit) in isoosmotic buffered saline, $\mathrm{pH}$ 7.0, were incubated at $37^{\circ} \mathrm{C}$ in media with different Nbd-Tau concentrations. Samples $(1 \mathrm{ml})$ were withdrawn and transferred to $30 \mathrm{ml}$ of pre-chilled $\left(4^{\circ} \mathrm{C}\right)$ medium, centrifuged, and the supernatant was discarded. Cell pellets were washed and centrifuged once again. The final pellets were resuspended in $1 \mathrm{ml}$ of distilled water to haemolyse the cells. An aliquot was used for the spectrophotometric determination of haemoglobin at $410 \mathrm{~nm}$. A second aliquot was deproteinized with trichloroacetic acid [5\% (w/v) final concn.] and the absorptivity of Nbd-Tau was read at $473 \mathrm{~nm}$. The haemoglobin content per unit of cell water space was determined as previously described (Cabantchik \& Ginsburg, 1977).

\section{Loading of erythrocytes with $\mathrm{Nbd-Tau}$}

Erythrocytes, either loaded with different $\mathrm{Cl}^{-}$ concentrations by the Amphotericin B method or washed with iso-osmotic buffered saline, $\mathrm{pH} 7.0$, were incubated with $5 \mathrm{~mm}$-Nbd-Tau (or as indicated), for $5-7 \mathrm{~h}$ at $37^{\circ} \mathrm{C}$ with gentle shaking. The final volume of solution was $0.3-1 \mathrm{ml}$ and the haematocrit was $10 \%$. The intracellular concentration of Nbd-Tau was determined as shown above.

\section{Loading of cells with ${ }^{36} \mathrm{Cl}^{-}$and $\mathrm{Cl}^{-}$fluxes}

Cells previously loaded with the appropriate $\mathrm{Cl}^{-}$ and/or Nbd-Tau concentrations were washed twice with the appropriate buffer (i.e. same $\mathrm{Cl}^{-}$concn. as under the loading conditions) at $0^{\circ} \mathrm{C}$ and incubated at $30 \%$ haematocrit with ${ }^{36} \mathrm{Cl}^{-}$(The Radiochemical Centre) $(0.3-1.0 \mu \mathrm{Ci} / \mathrm{ml})$ for $20 \mathrm{~min}$, at $4^{\circ} \mathrm{C}$. Aliquots (approx. $80 \mu \mathrm{l}$ ) were transferred to polyethylene tubes and centrifuged for $3 \mathrm{~min}$ in a Beckman Microfuge at $4^{\circ} \mathrm{C}$. The supernate was aspirated and the end of the tube was cut off. The cells were sucked into a short length of polyethylene tubing attached to a $10 \mathrm{ml}$ syringe pre-filled with $5 \mathrm{ml}$ of flux medium. Flux was started by flushing the cells with the above $5 \mathrm{ml}$ of medium into a jacketed vessel containing an additional $25 \mathrm{ml}$ of medium at constant stirring and constant temperature $\left(0^{\circ} \mathrm{C}\right)$ maintained with a Hetofrig controlled temperature circulator (Heto, Birkeröd, Denmark). Samples were withdrawn at appropriate intervals by aspirating $0.8-1.0 \mathrm{ml}$ through a combined prefilter $/ 1.2 \mu \mathrm{m}$ filter unit locked in $25 \mathrm{~mm}$ diam. holders (Sartorius $\mathrm{GmbH}$ ) and connected to $5 \mathrm{ml}$ Luer-lock syringes (Mawe \& Hempling, 1969; Dalmark \& Wieth, 1972). At the end of the experiment, $0.5 \mathrm{ml}$ of each filtrate (i.e. cell-free medium) was added to $0.1 \mathrm{ml}$ of aqueous trichloroacetic acid $(30 \%, w / v)$, centrifuged, and $0.4 \mathrm{ml}$ aliquots were transferred for counting of radioactivity with Hydroluma scintillation fluid ( $3.5 \mathrm{ml}$, Lumac AC). Samples were counted in a Packard PL scintillation counter. Similarly, $0.5 \mathrm{ml}$ of the total suspension (time infinity, $\infty$ ) were treated with trichloroacetic acid, centrifuged and counted.

For equilibrium exchange conditions, the isotope exchange is given by the equation (Gardos et al., 1969)

$$
\ln \left(\frac{C_{\infty}-C_{t}}{C_{\infty}}\right)=-k \cdot t
$$

where $C_{t}$ and $C_{\infty}$ are the radioactivities of ${ }^{36} \mathrm{Cl}^{-}$ appearing in the external medium at time $t$ and time infinity $(\infty)$ (i.e. time of full equilibration) respectively, and $k$ is the corresponding rate constant. Values of $k$ were obtained from the slope of the natural logarithm of the fractional radioactivity remaining in cells $\left\{\ln \left[\left(C_{\infty}-C_{t}\right) / C_{\infty}\right]\right\}$ plotted against time $t$ (Cabantchik \& Ginsburg, 1977). For $\mathrm{Cl}^{-}$fluxes studied in the presence of either extracellular or intracellular Nbd-Tau, the resulting rate constants of $\mathrm{Cl}^{-}$exchange are designated $k_{\mathrm{i}}$, as compared with $k_{0}$ which designates $k$ in the absence of inhibitors.

\section{Continuous monitoring of Nbd-Tau efflux by fluorescence}

Cells $(100 \mu \mathrm{l}, 10 \%$ haematocrit) loaded with Nbd-Tau at the appropriate $\mathrm{Cl}^{-}$concentrations were transferred to $1 \mathrm{ml}$ of ice-cold iso-osmotic sucrose buffered with $30 \mathrm{~mm}$-Tris/citrate and centrifuged for $10 \mathrm{~s}$ in an Eppendorf microcentrifuge. The cell pellet was washed with an additional $1 \mathrm{ml}$ of the sucrose medium, suspended in $100 \mu \mathrm{l}$ of ice cold medium, and filtered through a column $(0.5 \mathrm{ml})$ of Dowex AG1 (X8, 25-50 mesh, citrate form) at $0^{\circ} \mathrm{C}$. The suspensions of cells in ice-cold sucrose/citrate medium facilitates the complete and fast removal of 
extracellular permeating anions without causing any measurable salt leakage from cells. To initiate fluxes, a $50 \mu \mathrm{l}$ aliquot of filtrate (approx. $1.5 \times 10^{6}$ cells) was injected into a cuvette of the spectrofluorimeter containing $2 \mathrm{ml}$ of efflux medium at the indicated temperature. The course of Nbd-Tau egress from cells was monitored fluorimetrically either in a continuous mode on a single sample or in a discontinuous mode on four samples run in parallel in four different cuvettes. Settings were as follows: excitation wavelength, $475 \mathrm{~nm}$ ( $5 \mathrm{~nm}$ slit); emission wavelength, $540 \mathrm{~nm}(5-10 \mathrm{~nm}$ slits $)$. Details of the method are given elsewhere (Eidelman \& Cabantchik, 1980).

\section{Analysis of kinetic data}

All kinetic data were evaluated either by linear regression analysis (Dahlberg, 1948; Brownlee, $1965)$ or by non-linear regression analysis (Marquandt, 1963) using a Wang PCSII desk computer. Kinetic parameters are given with the calculated S.E.M.

\section{Results}

\section{Loading of $\mathrm{Nbd}$-Tau into cells}

Erythrocytes take up Nbd-Tau in a concentration-dependent manner, reaching transmembrane equilibrium of probe (Donnan equilibrium) after several hours of incubation at $37^{\circ} \mathrm{C}, \mathrm{pH} 7.0-7.4$ (Fig. 3). For extracellular concentrations lower than $5 \mathrm{mM}, 6-7 \mathrm{~h}$ of incubation were sufficient for attaining equilibrium. At higher concentrations, the requisite times of equilibration were very long. The results shown in Fig. 3 suggest that, in addition to back-flux of probe from cells, the time of equili-

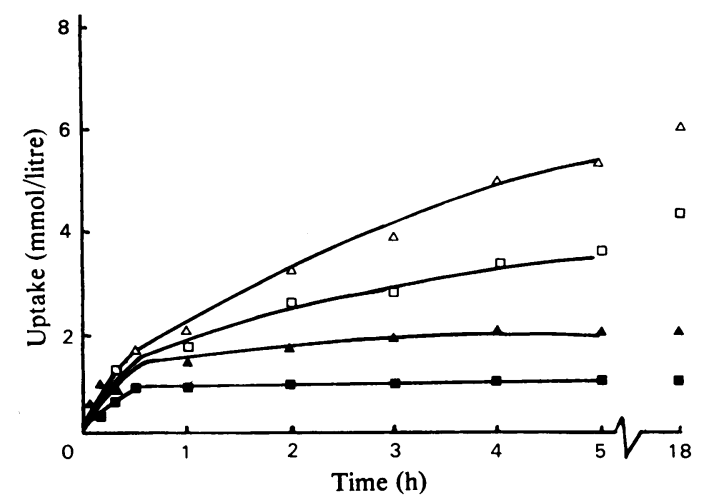

Fig. 3. Time course of Nbd-Tau uptake into intact erythrocytes

Uptake was measured at $37^{\circ} \mathrm{C}$ as described in the Materials and methods section. The concentrations of $\mathrm{Nbd}-\mathrm{Tau}$ in the loading medium were as follows:

$\mathbf{D}, 1 ; \Delta, 2 ; \square, 5$ and $\triangle, 10 \mathrm{~mm}$. bration is affected by a self-inhibiting effect of the probe on its own transport.

The probe taken up by the cells was chemically identical to original Nbd-Tau in spectroscopic as well as chromatographic properties. This indicates lack of deterioration or intracellular metabolic conversion of the probe in the above experimental conditions. Most of the intracellular Nbd-Tau $(>85 \%)$ was in the free form, as judged from the experimental Donnan ratios of $0.8-1.0$ found at $\mathrm{pH} 7.0-7.4\left(37^{\circ} \mathrm{C}\right)$. The probe was quantitatively retained in cells after washing the loaded cells with ice-cold medium. Dilution of the suspension of loaded cells with ice-cold medium and incubation for up to $20 \mathrm{~min}$ at $0^{\circ} \mathrm{C}$ resulted in no significant loss of intracellular material (results not shown). Similarly, no penetration of Nbd-Tau into cells was detected either at $0^{\circ} \mathrm{C}$ using untreated cells or at $37^{\circ} \mathrm{C}$ using cells chemically modified with the covalent reagent 4,4'-di-isothiocyano-2,2'-stilbene disulphonic acid (Cabantchik \& Rothstein, 1972).

\section{Inhibition of $\mathrm{Cl}^{-}$exchange by $\mathrm{Nbd}$-Tau}

In order to assess whether or not Nbd-Tau is a substrate of the anion-transport system, it needs to be shown that it inhibits competitively the transport of the natural substrate (i.e. $\mathrm{Cl}^{-}$) from either side of the membrane. Therefore, the inhibitory effects were measured on $\mathrm{Cl}^{-}$equilibrium exchange at $0^{\circ} \mathrm{C}$ with the probe present either inside or outside the cells. In these conditions (i.e. $0^{\circ} \mathrm{C}$ ), the permeation of Nbd-Tau through the erythrocyte membrane is minimal (see above), while that of $\mathrm{Cl}^{-}$is easily measurable.

The inhibition of $\mathrm{Cl}^{-}$exchange can occur either by interaction with $\mathrm{Cl}^{-}$transport sites, $\mathrm{Cl}^{-}$modifier sites, or non-specific sites (Dalmark, 1976; Cabantchik et al., 1978; Knauf, 1979). As shown before (Cabantchik et al., 1978; Barzilay \& Cabantchik, $1979 a$ ), the inhibition of $\mathrm{Cl}^{-}$exchange by interaction with a single class of sites can be described in terms of the modified Dixon plot (Webb, 1963):

$k_{0} / k_{\mathrm{i}}=1+\frac{[\mathrm{I}]}{K_{\mathrm{i}}^{\ln }(1+[\mathrm{S}] / K /)}$ for a compețitive inhibitor

and as $k_{0} / k_{\mathrm{i}}=1+[\mathrm{I}] / K_{\mathrm{i}}$ for a non-competitive inhibitor, where $k_{0}$ and $k_{\mathrm{i}}$ are the respective rate constants of $\mathrm{Cl}^{-}$efflux in the absence and in the presence of inhibitor, [I] and [S] are the concentrations of Nbd-Tau and $\mathrm{Cl}^{-}$respectively, $K$ is either the Michaelis constant for the transport site (i.e. $K_{\mathrm{s}}$ ) or the constant of inhibition of $\mathrm{Cl}^{-}$on its own transport via binding to modifier sites (i.e. $K_{\mathrm{s}}^{\prime}$ ), and $K_{\mathrm{i}}$ is the inhibition constant of Nbd-Tau. Thus, with the aid of the modified Dixon plot, it is possible to distinguish between competitive and non-competitive inhibition. Furthermore, for the case of 
competitive inhibition, a replot of the $x$-intercepts, which are equivalent to the apparent $K_{\mathrm{i}}\left(K_{\mathrm{i} \text {, app. }}\right)$

$$
K_{\mathrm{i}, \text { app. }}=K_{\mathrm{i}}(1+[\mathrm{S}] / K)
$$

against [S], allows the determination of $K_{1}$ as well as of $K$. The calculated $K$ value can be compared against the known values of $K_{\mathrm{s}}$ and $K_{\mathrm{s}}^{\prime}$, and thus the nature of the site of inhibition can be defined.

\section{Inhibition from inside}

In order to assess whether intracellular Nbd-Tau affects anion exchange, cells loaded with different $\mathrm{Cl}^{-}$concentrations were also loaded with Nbd-Tau to different intracellular concentrations. Extracellular Nbd-Tau was removed by centrifugation and washing, and subsequently ${ }^{36} \mathrm{Cl}^{-}$was incorporated by a $20 \mathrm{~min}$ incubation at $0^{\circ} \mathrm{C}$. Efflux was measured in an Nbd-Tau-free medium at $0^{\circ} \mathrm{C}$ containing $\mathrm{Cl}^{-}$at the same concentration as in the loading medium. The inhibition of $\mathrm{Cl}^{-}$exchange by Nbd-Tau is shown in Fig. 4 (upper) in terms of the modified Dixon plot, and in Fig. 4 (lower) as a replot of $K_{\mathrm{i} \text {,app. }}^{\text {in }}$ (the $x$-intercept of the Dixon plot as a function of $\left[\mathrm{Cl}^{-}\right]$. The variation of $K_{\mathrm{i} \text {,app. }}^{\text {in }}$ with $\left|\mathrm{Cl}^{-}\right|$is indicative of competitive inhibition. The extrapolated $K_{\mathrm{i} \text {, app. }}^{\text {in }}$ at $\left[\mathrm{Cl}^{-}\right]=0, K_{\mathrm{i}}^{\text {in }}$ is $3.5 \pm$ $1.5 \mathrm{~mm}$ and the value of $K$ is $53 \pm 34 \mathrm{~mm}$.

Since the $K_{\mathrm{s}}$ for $\mathrm{Cl}^{-}$exchange at $0^{\circ} \mathrm{C}$ is $65 \mathrm{~mm}$, whereas the $K_{\mathrm{x}}^{\prime}$ is $335 \mathrm{mM}$, it is suggested that Nbd-Tau competes with chloride for anion-transport sites at the internal surface of the membrane. Similar results were obtained with resealed 'ghosts', which had one-tenth the intracellular concentration of haemoglobin.

\section{Inhibition from outside}

The inhibitory effect of Nbd-Tau on $\mathrm{Cl}^{-}$exchange was considerably more pronounced from without than from within cells. A 200 -fold difference in $K_{i}^{\text {out }}$ versus $K_{i}^{\text {in }}$ indicates a marked asymmetry in the nature of the sites with which the probe interacts at the two surfaces. Regarding interactions at the outer surface, the modified Dixon plots for various $\left[\mathrm{Cl}^{-}\right]$(Fig. 5, upper part) were apparently linear over the range $0-0.1 \mathrm{~mm}-\mathrm{Nbd}-\mathrm{Tau}$, indicating that inhibition resulted primarily from interactions with a single class of inhibitory sites. These interactions were of an apparent competitive nature (Fig. 5, lower part), displaying a $K_{1}^{\text {out }}$ of $7.3 \pm 1.4 \mu \mathrm{M}$ and a $K$ of $165 \pm 60 \mathrm{mM}$. At this stage it would be premature to exclude interactions also with additional inhibitory sites, inasmuch as these appear not to be manifested as deviations from linearity in the above Dixon plots (Fig. 5, upper part). If these interactions in fact occur, we can predict that they are of low affinity nature, displaying $K_{1}$ values in the mM range. From present kinetic data it is not clear whether the main inhibitory effect of Nbd-Tau on
$\mathrm{Cl}^{-}$exchange resulted from interactions with either transport or modifier sites. The calculated $K$ value was significantly different $(P<0.001)$ from the $K_{\mathrm{s}}$ value of $65 \pm 5 \mathrm{mM}$ reported by Brazy \& Gunn (1976) or from the $K_{\mathrm{s}}^{\prime}$ value of $335 \mathrm{~mm}$ reported by Dalmark (1976). However, as pointed out by Knauf et al. (1979), the reported values were subject to considerable error due to the scattering of data as well as to the simplifications used in their calculations (Dalmark, 1976). Thus, the nature of the Nbd-Tau inhibitory sites cannot be specified.
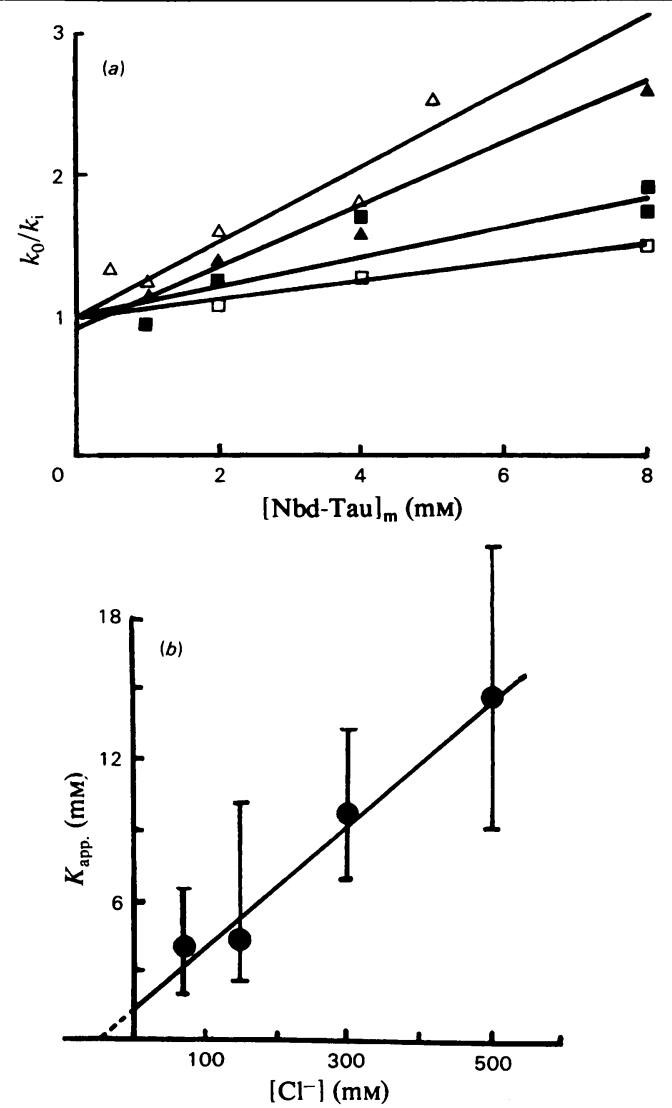

Fig. 4. Inhibition of $\mathrm{Cl}^{-}$exchange by internal Nbd-Tau Erythrocytes were equilibrated by the Amphotericin method to the following chloride concentrations: $\triangle$, $75 ; \Delta, 150 ; \square, 300$ and $\square, 500 \mathrm{~mm}$. The cells were subsequently loaded with Nbd-Tau to different final concentrations and incubated with $\mathrm{Na}^{36} \mathrm{Cl}$ for isotope equilibration. The efflux of ${ }^{36} \mathrm{Cl}^{-}$from cells into Nbd-Tau-free and radioisotope-free media was measured at $0^{\circ} \mathrm{C}$. The calculated rate constants of efflux in the presence and absence of inhibitor, are $k_{1}$ and $k_{0}$ respectively. Upper graph, relative Dixon plot of $k_{0} / k_{1}$ versus Nbd-Tau concentration; lower graph, the $x$-intercept values obtained from the above graph, $K_{\text {app. }}$, are plotted against $\mathrm{Cl}^{-}$concentration. The intercept on the $y$-axis yields $K_{1}=3.5 \pm 1.5 \mathrm{mM}$ and the intercept on the $x$-axis yields $K=53 \pm 34 \mathrm{~mm}\left(r^{2}=0.98\right)$. Bars denote S.D. values. 

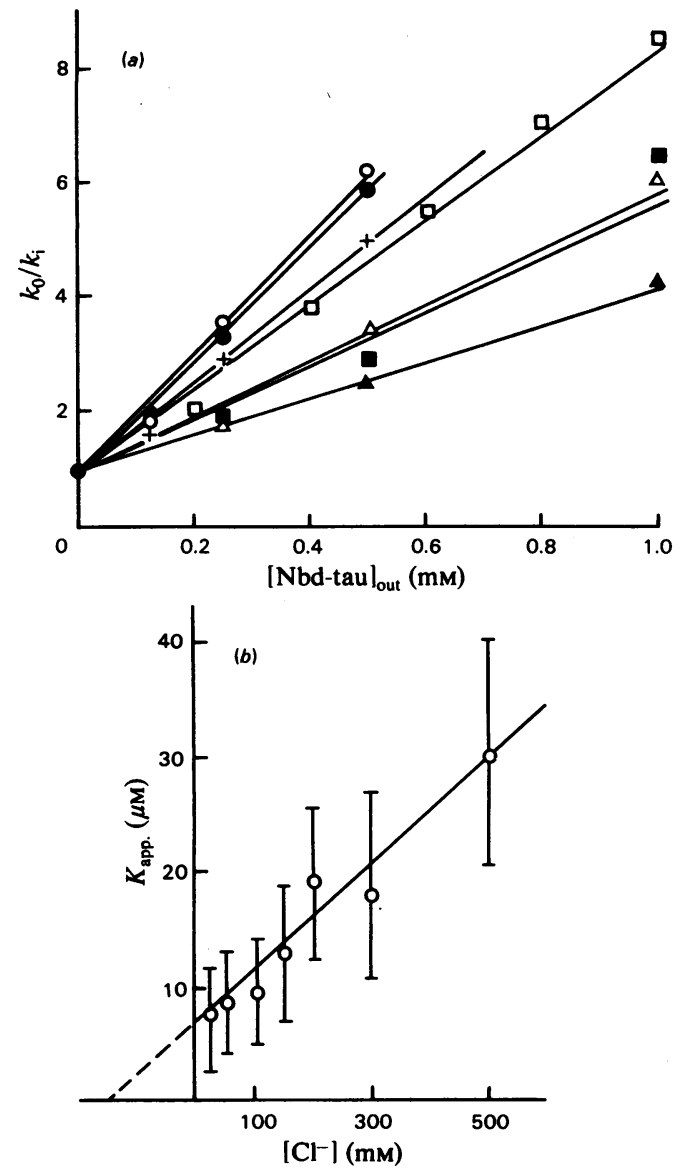

Fig. 5. Inhibition of $\mathrm{Cl}^{-}$exchange by external $\mathrm{Nbd}-\mathrm{Tau}$ Cells were loaded with different $\mathrm{Cl}^{-}$concentrations and equilibrated with ${ }^{36} \mathrm{Cl}$ as described in Fig. 3 . Efflux was measured at $0^{\circ} \mathrm{C}$ into media containing different Nbd-Tau concentrations. The upper graph is the modified Dixon plot of $k_{0} / k_{\mathrm{i}}$ versus $\mathrm{Nbd}$-Tau concentration obtained at different $\mathrm{Cl}^{-}$concentrations. Symbols are as follows (in $\mathrm{mm}$ ): (O) 25 ,

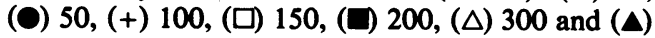
500. The $K_{\mathrm{i} \text {, app. }}$ calculated for the various systems as shown in Fig. 4 were plotted against the $\mathrm{Cl}^{-}$ concentration (lower graph) yielding the following parameters: $K_{1}=7.3 \pm 1.4 \mu \mathrm{M}$ and $K=165 \pm 60 \mathrm{mM}$ $\left(r^{2}=0.97\right)$.

Continuous monitoring of anion transport by fluorescence: Nbd-Tau efflux from cells and its susceptibility to inhibitors of anion transport

The efflux of Nbd-Tau was followed in a spectrofluorimeter by continuous monitoring of the fluorescence of a suspension of cells loaded with probe without separation of medium from cells (Fig. 6). The instantaneous rise in fluorescence after addition of cells $[F(0)]$ stems primarily from scattering of light by cells (intracellular fluorescence is mostly quenched by haemoglobin). It is followed by a time-dependent increase in fluorescence originating from Nbd-Tau molecules that have emerged from cells. Evidence for the latter is provided by a parellel experiment in which cellfree supernatants obtainèd by filtration were measured for fluorescence at indicated times (filled circles in Fig. 6). When the fluorescence reaches a plateau level (i.e. at equilibrium) $F(\infty)$, an aliquot of concentrated Nbd-Tau was added to the cell suspension in order to calibrate the fluorescence intensity scale. Fluorescence was linear up to $2 \mu \mathrm{M}-\mathrm{Nbd}$-Tau. In order to prevent self-inhibition, flux experiments were run always under conditions in which extracellular Nbd-Tau at $t=\infty$ never exceeded $0.5 \mu \mathrm{M}$.

The rate constant $(k)$ of Nbd-Tau efflux was obtained by taking at least 20 experimental points along the fluorescence tracing (Fig. 6) and fitting them to the exponential relationship:

$$
F(t)=F(\infty)-[F(\infty)-F(0)] \exp (-k t)
$$

by a non-linear regression program based on the Lavenberg-Marquardt algorithm (Marquardt, 1963). The various $F$ values represent the fluorescence intensity at the respective times $t, 0$ and $\infty$. The non-linear least squares analysis gave an exponential fit virtually indistinguishable from the experimental tracing $\left(k=0.061 \pm 0.001, r^{2}=0.99\right)$. The corresponding $k$ for data obtained with the filtration method was $0.062 \pm 0.002\left(r^{2}=0.98\right)$. The efflux profile of Nbd-Tau was virtually the same in media containing any of these buffers (at $20 \mathrm{~mm}$ ): phosphate, citrate, Hepes[4-( $N$-2-hydroxyethyl)-1piperazine-ethanesulphonic acid], Tris, and in media containing any of these cations: $\mathrm{Na}^{+}, \mathrm{K}^{+}$, choline or Tris.

The method of monitoring transport by fluorescence was particularly useful for screening the effects of inhibitors (or other factors) which affect anion transport. Traces of fluxes of four different systems run in parallel are shown in Fig. 7. Although the fluxes were monitored discontinuously, they were sufficiently informative for calculating the requisite kinetic parameters of Nbd-Tau transport. These were obtained for two types of inhibitors, DNDS, a specific, non-penetrating inhibitor, and phloretin, a dipole potential modifier (Deuticke, 1977). Both compounds gave linear inhibition profiles to at least 95\% inhibition) in the modified Dixon plots (Fig. 8, inserts), yielding $K_{1 \text {,app. }}$ of $13.9 \pm 6.6 \mu \mathrm{M}$ for DNDS and $7.1 \pm 0.5 \mu \mathrm{M}$ for phloretin. These values are comparable with those observed for inhibition of either $\mathrm{Cl}^{-}$exchange or sulphate exchange (Deuticke, 1977; Cabantchik et al., 1978; Knauf, 1979). Nbd-Tau fluxes were also sensitive to chemical 


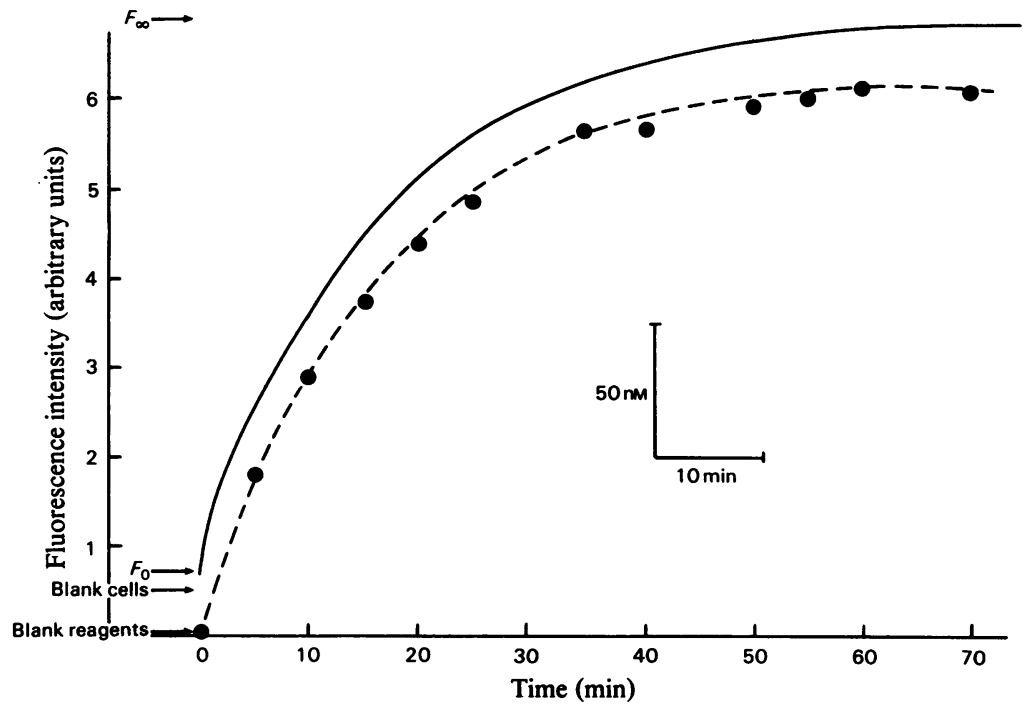

Fig. 6. Efflux of Nbd-Tau from cells

Cells loaded with Nbd-Tau ( $4 \mathrm{mM}$ ) and filtered at $0^{\circ} \mathrm{C}$ through a Dowex AG 1 (X8; citrate form) minicolumn were injected into a thermostated vessel containing $25 \mathrm{ml}$ of phosphate-buffered saline $(\mathrm{pH} 7.0)$, at $37^{\circ} \mathrm{C}$, to give $1 \times 10^{6}$ cells $/ \mathrm{ml}$ of suspension. A $2 \mathrm{ml}$ aliquot of suspension was quickly transferred to a cuvette in the spectrofluorimeter, and the fluorescence intensity was followed at $37^{\circ} \mathrm{C}$ in a continuous fashion (-). In parallel, samples were withdrawn from the vessel, filtered through Sartorius filters (1.2 $\mu \mathrm{m}$ mean pore diameter) as described in the Materials and methods section, and the fluorescence intensity of the filtrates were measured (0). Data analysed by the non-linear least squares method gave the following rate constants: $k=0.061 \pm 0.001\left(r^{2}=0.99\right)$ for the method of continuous monitoring and $k=0.062 \pm 0.002\left(r^{2}=0.98\right)$ for the method of sampling.
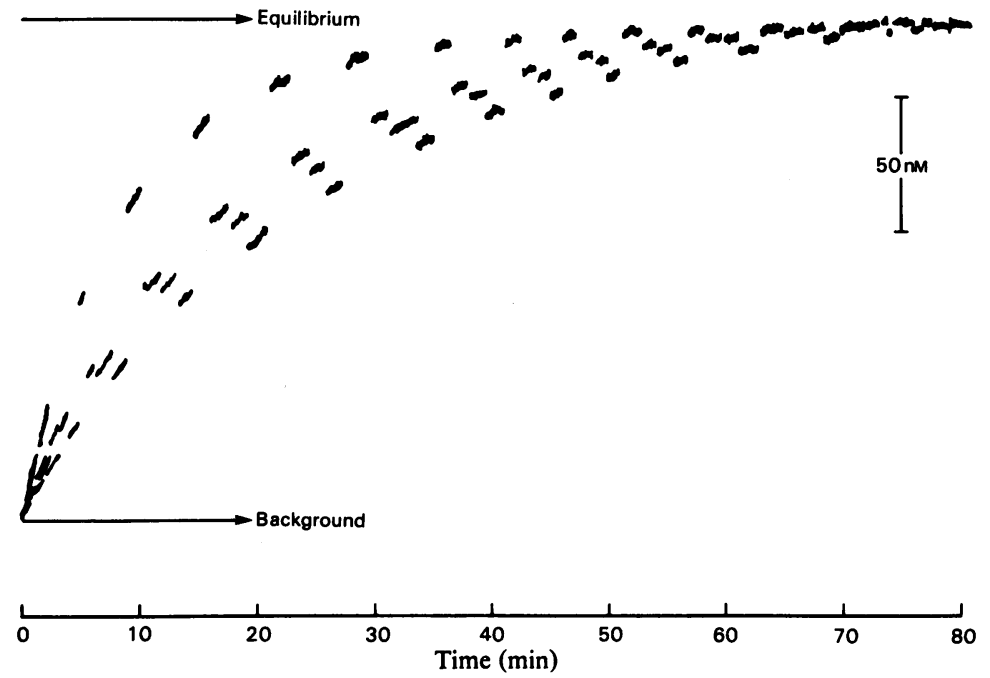

Fig. 7. Comparative efflux profiles of Nbd-Tau

The efflux of Nbd-Tau from cells (see Fig. 5) was fluorimetrically measured at $37^{\circ} \mathrm{C}$ in Tris/saline buffered media (pH 7.0), containing either $0,10,15$ or $20 \mu \mathrm{M}$-DNDS.The discontinuous traces of four parallel systems were obtained by rotating the multicuvette turret at given time intervals. All systems contained the same number of cells $\left(4 \times 10^{5} / \mathrm{ml}\right)$. 

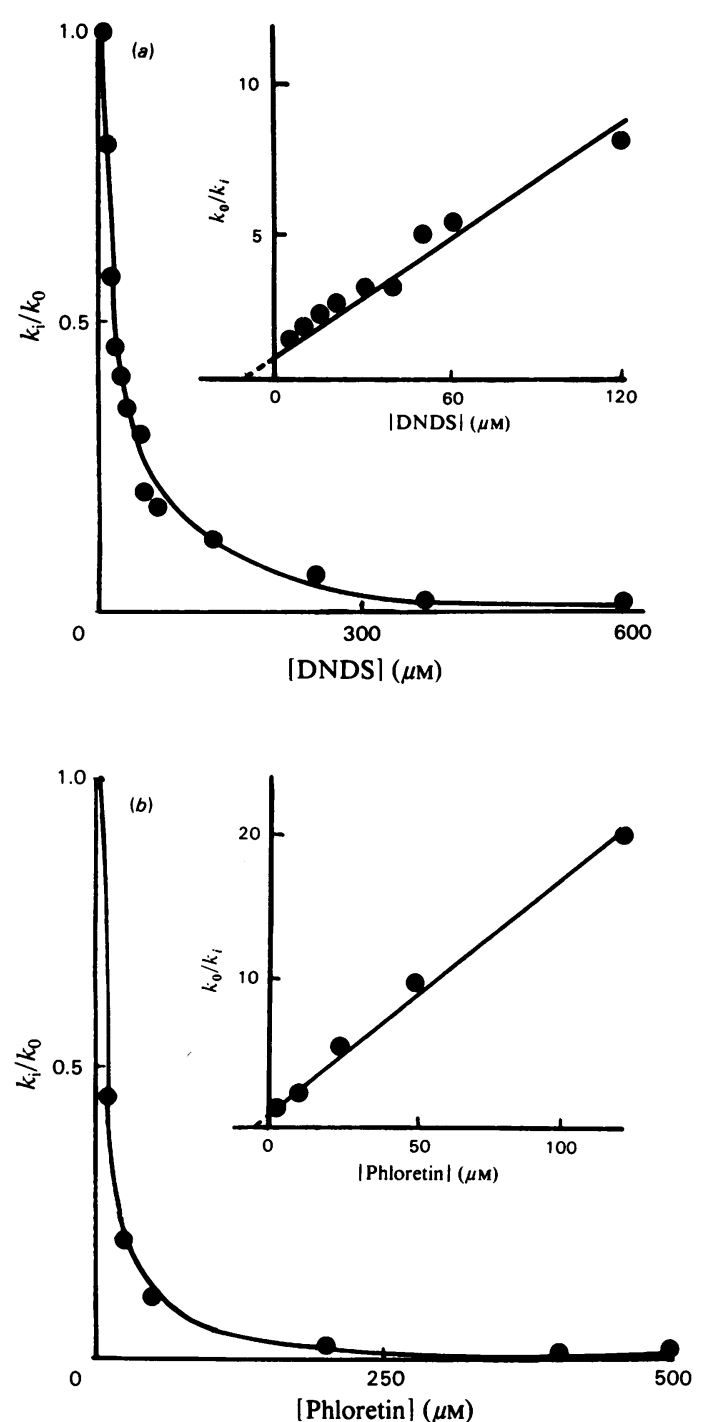

Fig. 8. Inhibitory effects of DNDS and phloretin on Nbd-Tau efflux

Nbd-Tau efflux was measured spectrofluorimetrically in media containing different concentrations of inhibitors [I]. The fractional flux $k_{\mathrm{i}} / k_{0}$ is plotted against the corresponding concentration of inhibitor. The modified Dixon plots (see Fig. 4) given in the insets were constructed from same data but for ranges of [I] where $k_{0} / k_{1}$ values were lower than 20 (i.e., 95\% inhibition). Linear regression analysis of the linearized plots gave the following $K_{\mathrm{i} \text {, app. }}(\mu \mathrm{M})$ values: $13.9 \pm 6.6$ for DNDS and $7.1 \pm 9.5$ for phloretin.

modification of cells by $4,4^{\prime}$-di-isothiocyano- $2,2^{\prime}$ stilbene disulphonic acid, a covalent reacting analgoue of DNDS (Cabantchik \& Rothstein, 1972) which binds irreversibly to the anion transporter (results not shown).
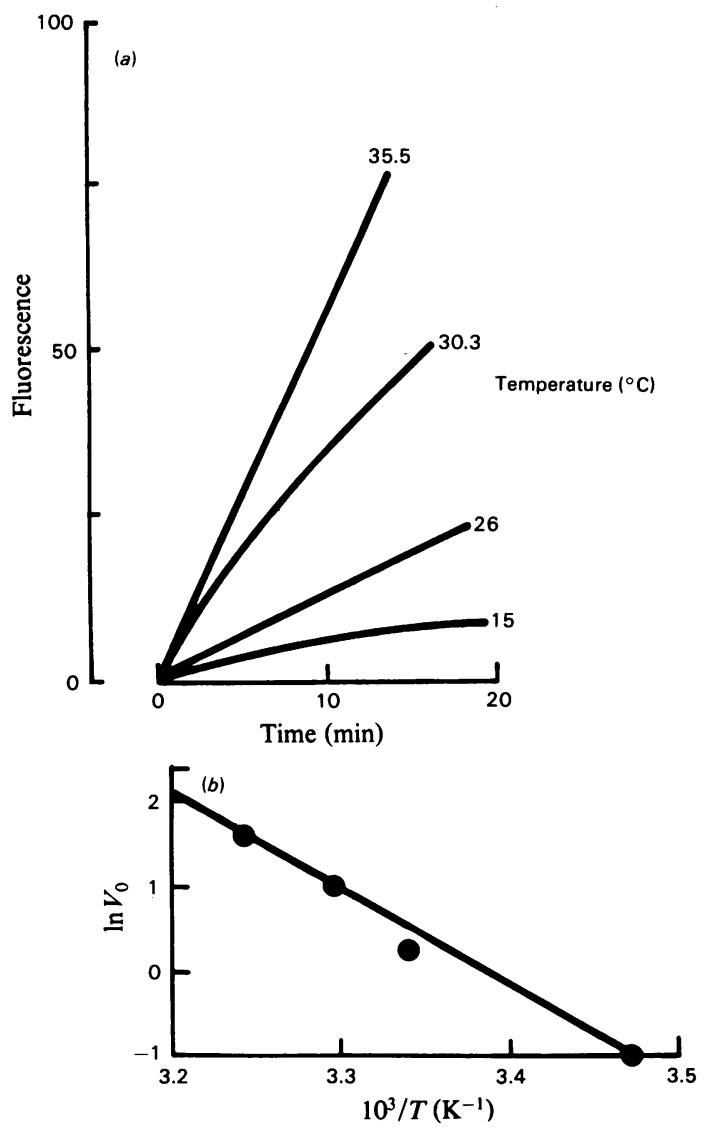

Fig. 9. Temperature dependence of Nbd-Tau efflux Efflux of Nbd-Tau from cells (Fig. 6) was monitored at different temperatures in phosphatebuffered saline ( $\mathrm{pH}$ 7.4) (upper graph). The calculated initial rates normalized to the same number of cells were plotted according to the Arrhenius scheme. The energy of activation calculated was $23.4 \pm 1.4 \mathrm{kcal} / \mathrm{mol}(98 \pm 6 \mathrm{~kJ} / \mathrm{mol})$.

Effect of temperature and of $\mathrm{Cl}^{-}$concentration on Nbd-Tau efflux

Like halide and sulphate exchange, Nbd-Tau efflux was temperature-dependent (Fig. 9). An Arrhenius plot constructed from efflux profiles obtained in the $15-35.5^{\circ} \mathrm{C}$ range gave an energy of activation of $23.4 \pm 1.4 \mathrm{kcal} / \mathrm{mol}(98 \pm 6 \mathrm{~kJ} / \mathrm{mol})$, This value is similar to that obtained for $\mathrm{Cl}^{-}$ exchange (Brahm, 1977) and is less than that described for sulphate (Passow, 1969).

The effect of $\mathrm{Cl}^{-}$on Nbd-Tau efflux was measured in cells loaded with different $\mathrm{Cl}^{-}$concentrations, using the Amphotericin technique. Most of the Amphotericin was apparently removed from the cells by washing, inasmuch as Nbd-Tau efflux was virtually the same in Amphotericin-treated or untreated cells. The symmetrical increase in $\mathrm{Cl}^{-}$ 


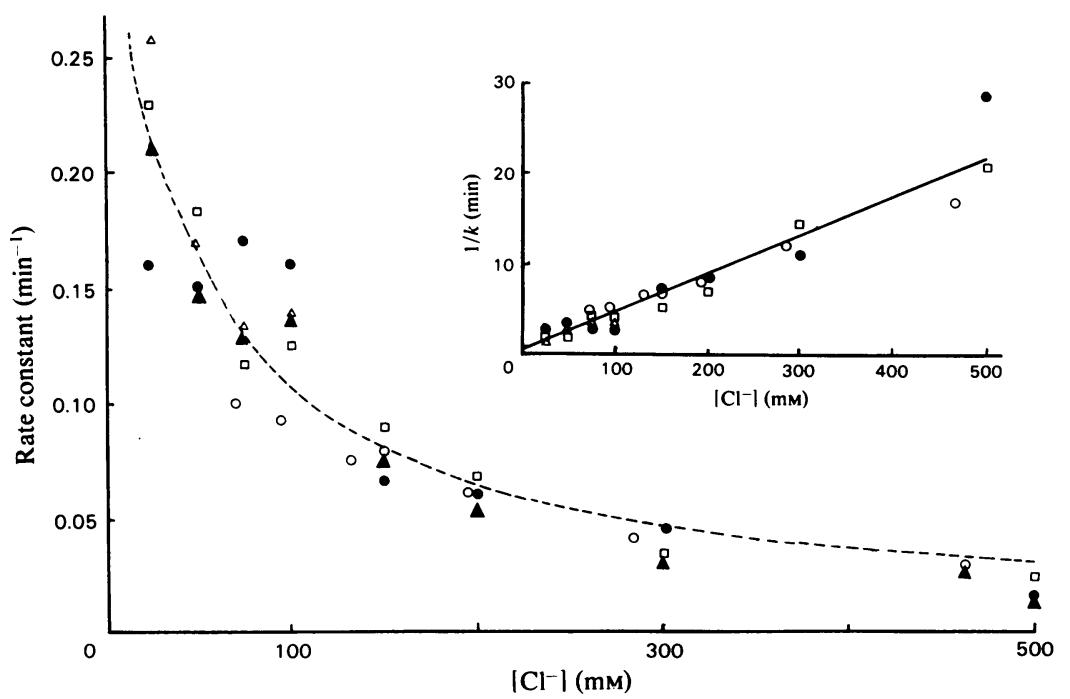

Fig. 10. Effect of $\mathrm{Cl}^{-}$concentration on Nbd-Tau efflux

Cells were loaded with $\mathrm{Cl}^{-}$to the indicated concentrations and with Nbd-Tau (4 mM) in phosphate-buffered medium (pH 7.4) (see Fig. 4). The cells were washed of external probe and jetted into media containing the same $\mathrm{Cl}^{-}$ concentrations as in the loading media. The rate constant $(k)$ of Nbd-Tau efflux is plotted against the $\mathrm{Cl}^{-}$ concentration. The inset describes the Dixon plot of the same data. $O, \mathbf{\square}, \Delta$ and $\bullet$ denote ranges of rate constants obtained in four separate experiments; ( $\mathbf{\Delta})$ denotes rate constants normalized for volume changes observed in cells at $\mathrm{Cl}^{-}$concentrations different from $150 \mathrm{~mm}$. The lines connecting through the points are the best fits obtained either by non-linear least squares method (broken line), which yielded $K_{1}=49 \pm 12 \mathrm{mM}\left(r^{2}=0.87\right)$ for $\mathrm{Cl}^{-}$inhibition of Nbd-Tau efflux, or by the linear least-squares method (full line, inset) which yielded $K_{\mathrm{i}, \text { app. }}=15 \pm 12 \mathrm{mM}\left(r^{2}=0.92\right)$.

concentration outside and inside the cell produced a progressive inhibition of Nbd-Tau efflux (Fig. 10). Analysis of this inhibition, either by non-linear least squares analysis of $k$ versus $\left[\mathrm{Cl}^{-}\right]$or by linear least squares analysis of $1 / k$ versus $\left[\mathrm{Cl}^{-}\right]$(i.e. a Dixon plot) (Fig. 10, insert), showed that, at $37^{\circ} \mathrm{C}, ~^{-} \mathrm{Cl}^{-}$ inhibited Nbd-Tau efflux by interaction with a single class of sites whose $K_{\mathrm{f}}$ was $49 \pm 12 \mathrm{mM}\left(r^{2}=0.87\right)$. Since this value is comparable with the $K_{\mathrm{s}}$ observed for $\mathrm{Cl}^{-}$transport at $37^{\circ} \mathrm{C}$ by $\mathrm{Brahm}$ (1977), we conclude that $\mathrm{Nbd}-\mathrm{Tau}$ and $\mathrm{Cl}^{-}$share a common transport site at the inner surface of the membrane.

\section{Discussion}

In the present work we introduce Nbd-Tau as a fluorescent substrate of the anion-transport system and the method of continuous monitoring of anion transport by fluorescence, which rests on the use of the above fluorescent substrate.

The selection of the fluorescent nitrophenyldiazole derivative of taurine (Nbd-Tau) as a potential substrate of the anion transporter was based primarily on the previous work of Cabantchik et al. (1976) and Knauf et al. (1978), who showed that the 2-nitrophenyl-4-azidophenyl derivative of taur- ine could serve as a substrate of the system. The two compounds were found to share structural elements which in previous structure-activity relationship studies have been shown to be essential for the interaction of various organic anions with the anion transporter, that are an anionic moiety and an aromatic hydrophobic counterpart carrying chemical groups of demonstrable $\mathrm{e}^{-}$-acceptor capacity (Barzilay et al., 1979). Specifically, the compounds share the tauryl group on the one hand, and the phenyl moiety substituted either with nitro, diazole or azido groups, on the other hand. Support for the rationale was found in preliminary screening studies which led to the finding that the 2,4dinitrophenyl derivative of taurine could also serve as a substrate of the system (D. Jones \& Z. I. Cabantchik, unpublished work) and to the finding that the fluorescent nitrophenyldiazole moiety showed marked $\mathrm{e}^{-}$-acceptor potential (Lancet \& Pecht, 1977). The Nbd-group was selected primarily because its excitation and emission spectra overlap largely with the absorption spectrum of haemoglobin. This ensures that in dilute cell suspensions fluorescence originating from intracellular Nbd-Tau will be mostly quenched by the absorbing haemoglobin, whereas fluorescence from extracellular Nbd-Tau will be minimally quenched. Since 
Nbd-Tau shows very limited permeation across red cell membranes at $0^{\circ} \mathrm{C}$, it was possible to load cells with the probe at $37^{\circ} \mathrm{C}$, separate loaded cells from the extracellular probe at $0^{\circ} \mathrm{C}$, and follow the egress from cells at $37^{\circ} \mathrm{C}$ into probe-free media. This egress was recorded fluorimetrically as a progressive increase in signal (Fig. 6). In that manner transport was monitored in a continuous fashion, circumventing the need for stopping transport, separating cells from medium and processing for counting for radioactivity at different time intervals.

In this work we attempted to demonstrate that Nbd-Tau can serve specifically as a substrate of the anion-transport system of human erythrocytes cells. First, we showed that under conditions of minimal penetration (i.e. at $0^{\circ} \mathrm{C}$ ), $\mathrm{Nbd}-\mathrm{Tau}$ placed intracellularly inhibited $\mathrm{Cl}^{-}$exchange in a competitive fashion (Fig. 4). The site of inhibition was apparently the $\mathrm{Cl}^{-}$transport site, as judged by the similar values of $K$ obtained from competition kinetics $(53 \pm 34 \mathrm{~m})$ (Fig. 4) and the reported $\mathrm{K}_{\mathrm{s}}$ of $\mathrm{Cl}^{-}$ exchange of $65 \pm 5 \mathrm{~mm}$ (Brazy \& Gunn, 1976). These results are analogous to those reported for the 2-nitro-4-azidophenyl derivative of taurine, which also interacts with anion-transport sites at the inner membrane surface (Knauf et al., 1978).

Secondly, Nbd-Tau efflux, as measured spectrofluorimetrically, was fully sensitive to classical inhibitors of anion exchange such as DNDS and phloretin (Fig. 8), and the bimodal reagents 4,4' di-isothiocyano-2,2'-stilbene disulphonic acid and its dihydro analog, to the diuretics furosemide and MK-196 (Merck, Sharp \& Dohm), and to the anti-inflammatory niflumic acid (results not shown). This reinforces the view that Nbd-Tau exits the cells via the anion transporter of erythrocytes. However, the observed $K_{1, \text { app }}$ of DNDS on Nbd-Tau efflux (measured at $37^{\circ} \mathrm{C}$ ) was $4-6$-fold higher than the $K_{1, \text { app }}$ on $\mathrm{SO}_{4}^{2-}$ exchange (measured at $25^{\circ} \mathrm{C}$ ) (Barzilay \& Cabantchik, 1979a) or was 1.5 -fold higher than $K_{\mathrm{i} \text {, app. }}$ on $\mathrm{Cl}^{-}$exchange (measured at $0^{\circ} \mathrm{C}$ ) (Barzilay \& Cabantchik, 1979b; M. Barzilay \& Z. I. Cabantchik, unpublished work). This apparent discrepancy in the susceptibility of the transport system to a particular inhibitor might reflect a fundamental difference in the transport mechanism of the various anions; however, it might likely reflect, at least in part, the temperature coefficient of the inhibitor effect. Such negative temperature coefficient for $K_{1 \text {, app. }}$ is compatible with the recent work of Cousin \& Motais (1979) which shows that the inhibitory potency of niflumic acid on anion exchange was reduced 10 -fold when the temperature was raised from $0^{\circ} \mathrm{C}$ to $30^{\circ} \mathrm{C}$.

Thirdly, the temperature dependence of Nbd-Tau efflux (range $15-35.5^{\circ} \mathrm{C}$ ) gave an activation energy of $23.4 \pm 1.4 \mathrm{kcal} / \mathrm{mol}(98 \pm 6 \mathrm{~kJ} / \mathrm{mol})$. This value is similar to that published for $\mathrm{Cl}^{-}$exchange measured in the same range of temperatures (Brahm, 1977), but it is about 30\% lower than the activation energy of sulphate or oxalate exchange (Passow, 1969; Cousin \& Motais, 1979). The cause of these differences needs to be explored further.

Finally, the most important criterion on which the present work rests is that of competition between Nbd-Tau and $\mathrm{Cl}^{-}$for common transport sites at the inner membrane surface. From the kinetic analysis of Nbd-Tau fluxes measured at different chloride concentrations we obtain that the $K_{\mathrm{i} \text {, app }}$ of $\mathrm{Cl}^{-}$is $49 \pm 12 \mathrm{mM}$, a value not significantly different from that reported for $\mathrm{Cl}^{-}$exchange (Dalmark, 1976; Brazy \& Gunn, 1976; Gunn \& Frohlich, 1979). Since the $K_{\mathrm{s}}$ for $\mathrm{Cl}^{-}$varies little with temperature, and since modifier effects at $37^{\circ} \mathrm{C}$ are very small up to $300 \mathrm{mM}^{-\mathrm{Cl}^{-}}$(Brahm, 1977), it would be safe to conclude that the competitive inhibition of $\mathrm{Cl}^{-}$on Nbd-Tau efflux resulted from interactions with $\mathrm{Cl}^{-}$ transport sites. Taken together, the above kinetic evidence is consistent with the idea that intracellular Nbd-Tau is transported via, if not exclusively by, the anion transport system of human erythrocytes.

The interactions of Nbd-Tau with the anion transport system at the two membrane surfaces are markedly asymmetric: at the inner surface, it acts on transport sites with $\mathrm{mM}$ apparent affinity (Fig. 4), but on the outer surface it acts on undefined sites with $\mu \mathrm{M}$ affinities (Fig. 5). These asymmetric effects are also reflected in the transport properties of Nbd-Tau which show much higher transport rates from inside than from outside (Figs. 3 and 6). A possible explanation for this observed asymmetry is that Nbd-Tau interacts with transport sites on both surfaces, inasmuch as it is transported in both directions but it also interacts with high affinity sites at the outer surface. This latter interaction might also lead to self-inhibition of Nbd-Tau uptake, as observed in the loading experiments (Fig. 3).

Unfortunately, the kinetic nature of Nbd-Tau inhibitory sites could not be resolved in the present series of studies. The fluorescent Nbd-Tau is chemically similar to the photochemically reactive 2-nitro-4-azidophenyl derivative of taurine, which has also been shown to interact rather specifically with the anion transport system of erythrocytes (Cabantchik et al., 1976; Rothstein et al., 1977). In a recent study, Knauf et al. (1978) have shown that the probe had a marked asymmetric effect on $\mathrm{Cl}^{-}$ exchange. This effect was attributed to the fact that the probe interacted competitively with transport sites at the inner surface, showing affinity at the $\mathrm{mm}$ range, but interacted with 'modifier' sites at the external surface, showing approx. 20-fold higher affinity. The two probes also show similar kinetics in their inward and outward transport rates (Knauf et al., 1978).

Based on the spectral properties of Nbd-Tau and 
of the erythrocyte system, as well as on present evidence that Nbd-Tau is a substrate of the anion-transport system, we are confident that the method of continuous monitoring of transport by fluorescence can be used as a convenient indicator of anion transport in intact cells. The method, which is sensitive down to $10^{5} \mathrm{cells} / \mathrm{ml}$ with normal instruments, is able to resolve fluxes in the minute range with standard mixing techniques and in the second range with appropriate rapid mixing devices. We have recently demonstrated (Darmon et al., 1980) that the method is also applicable to anion transport measurements in white 'ghosts' and in isolated, erythrocyte-derived, vesicles which contain no haemoglobin. In this case, however, purified anti(Nbd-albumin) antibodies were used to quench Nbd-Tau fluorescence in the outer medium.

At the present time it is not clear whether this method can be used to study anion transport in other types of cells. Nevertheless, with appropriate design of fluorescent analogues of substrates, it should be possible to apply the method of continuous monitoring of transport by fluorescence to study other transport systems of erythrocyte membranes (e.g., nucleoside, glucose) and hopefully of other cell membranes as well.

\section{Note Added in Proof (Received 13 March 1981)}

Recent studies (O. Eidelman, M. Zangvill \& Z. I. Cabantchik, unpublished work) have shown that the competitive inhibitor DNDS was approx. 6-fold more potent on Ndb-Tau transfer in sulphate medium than in chloride medium (both measured at $30^{\circ} \mathrm{C}, \mathrm{pH} 7.0$ ). These results are in line with previous observations made on DNDS inhibition of inorganic anion self-exchange (Barzilay \& Cabantchik, 1979a).

This work was supported in part by grants from the United States-Israel Binational Science Foundation and by the National Institutes of Health grant no. GM 27753.

\section{References}

Barzilay, M. \& Cabantchik, Z. I. (1979a) Membr. Biochem. 2, 255-281

Barzilay, M. \& Cabantchik, Z. I. (1979b) Membr. Biochem. 2, 297-321

Barzilay, M., Ship, S. \& Cabantchik, Z. I. (1979) Membr. Biochem. 2, 227-254

Brahm, J. (1977) J. Gen. Physiol. 70, 283-306

Brazy, P. C. \& Gunn, R. B. (1976) J. Gen. Physiol. 68, 583-599

Brownlee, K. A. (1965) Statistical Theory and Methodology, John Wiley \& Sons, Inc., New York

Cabantchik, Z. I. \& Ginsburg, H. (1977) J. Gen. Physiol. 69, 75-96
Cabantchik, Z. I. \& Loyter, A. (1980) Membr. Transport Erythrocytes, Alfred Benzon Symp. 14, 371-383

Cabantchik, Z. I. \& Rothstein, A. (1972) J. Membr. Biol. 10, 311-330

Cabantchik, Z. I., Knauf, P. A., Ostwald, T., Markus, H., Davidson, L., Breuer, W. \& Rothstein, A. (1976) Biochim. Biophys. Acta 455, 526-537

Cabantchik, Z. I., Knauf, P. A. \& Rothstein, A. (1978) Biochim. Biophys. Acta 515, 239-302

Cabantchik, Z. I., Volsky, D. J., Ginsburg, H. \& Loyter, A. (1980) Ann. N.Y. Acad. Sci. 341, 444-454

Cousin, J. L. \& Motais, R. (1979) J. Membr. Biol. 46, 125-153

Dahlberg, G. (1948) Statistical Methods for Medical and Biological Students. George Allen \& Unwin, London

Dalmark, M. (1976) J. Gen. Physiol. 67, 223-234

Dalmark, M. \& Wieth, J. O. (1972) J. Physiol. (Lond.) 244, 583-610

Darmon, A., Eidelman, O. \& Cabantchik, Z. I. (1980) Proc. FEBS Meet. 13th 23

Deuticke, B. (1977) Rev. Physiol. Biochem. Pharmacol. 78, 1-97

Eidelman, O. \& Cabantchik, Z. I. (1980) Anal. Biochem. 106, 335-342

Gardos, G., Hoffman, J. \& Passow, H. (1969) in Laboratory Techniques in Membrane Biophysics: an Introductory Course (Passow, H. \& Stampfli, R., eds.), pp. 9-20, Springer-Verlag, Berlin

Guidotti, G. (1980) in Membr. Transport Erythrocytes, Alfred Benzon Symp. 14, 300-308

Gunn, R. B. (1979) in Transport Across Biological Membranes (Giebisch, G., Tosteson, D. C. \& Ussing, H. H., eds.), pp. 59-80, Springer-Verlag, New York

Gunn, R. B. \& Fröhlich, O. (1979) J. Gen. Physiol. 74, 351-374

Knauf, P. A. (1979) Curr. Topics Membr. Transport 12, 249-363

Knauf, P. A., Ship, S., Breuer, W., McCulloch, L. \& Rothstein, A. (1978) J. Gen. Physiol. 72, 607-630

Lancet, O. D. \& Pecht, I. (1977) Biochemistry 16, 5150 5157

Marquardt, D. W. (1963) J. Soc. Industr. \& Appl. Math. 2, 431

Mawe, R. C. \& Hempling, H. G. (1965) J. Cell Comp. Physiol. 66, 95-103

Passow, H. (1969) Progr. Biophys. Mol. Biol. 19, 425-467

Ross, A. H. \& McConnell, H. M. (1978) J. Biol. Chem. 253, 4777-4782

Rothstein, A., Cabantchik, Z. I., Balshin, M. \& Juliano, R. L. (1975) Biochem. Biophys. Res. Commun. 64, 144-149

Schnell, K. F., Gerhardt, S. \& Schoppe-Fredenburg, A. (1977) J. Membr. Biol. 30, 319-350

Steck, T. L. (1978) J. Supramol. Struct. 8, 311-324

Tanner, M. J. A. (1978) Curr. Topics Membr. Transport 11, 279-325

Webb, J. L. (1963) Enzyme and Metabolic Inhibitors, vol. 1 , Academic Press, New York

Wieth, J. D. \& Brahm, J. (1980) in Membr. Transport Erythrocytes, Alfred Benzon Symp. 14, 467-482

Wolosin, J. M., Ginsburg, H. \& Cabantchik, Z. I. (1977) J. Biol. Chem. 252, 2419-2427 